\title{
Long non-coding RNA MEG3 inhibits cell migration and invasion of non-small cell lung cancer cells by regulating the miR-21-5p/PTEN axis
}

\author{
DONGJIN LV ${ }^{1}$, QING BI $^{1}$, YUNXIA LI ${ }^{1}$, JIE DENG $^{2}$, NA WU $^{1}$, SHU HAO $^{1}$ and MINGLI ZHAO ${ }^{1}$ \\ Departments of ${ }^{1}$ Medical Oncology and ${ }^{2}$ Pharmacy, The Third Affiliated Hospital of Kunming Medical University \\ (Tumor Hospital of Yunnan Province), Kunming, Yunnan 650118, P.R. China
}

Received March 16, 2020; Accepted October 15, 2020

DOI: $10.3892 / \mathrm{mmr} .2021 .11830$

\begin{abstract}
Long non-coding RNAs (lncRNAs) are involved in the occurrence and progression of numerous types of cancer. The aim of the present study was to evaluate the effect of the lncRNA maternally expressed gene 3 (MEG3) on the migration and invasion of non-small cell lung cancer (NSCLC) H1299 and PC9 cells. Reverse transcription-quantitative (RT-q)PCR analysis showed that MEG3 was downregulated in NSCLC PC9 and H1299 cells. Additionally, bioinformatics analysis indicated that MEG3 sponges microRNA (miR)-21-5p; miR-21-5p was predicted to target the phosphatase and tensin homolog (PTEN) 3'-untranslated region sequence. MEG3 overexpression led to miR-21-5p suppression and PTEN upregulation in PC9 and H1299 cells, as detected by RT-qPCR. Subsequently, western blot analysis confirmed that MEG3 overexpression enhanced PTEN expression levels and inhibited the PI3K/AKT signaling pathway in NSCLC cells. These effects were attenuated by miR-21-5p. Dual luciferase assay supported the sponging effect of MEG3 on miR-21-5p and validated the direct interaction between miR-21-5p and PTEN. Furthermore, Transwell assay demonstrated that MEG3 overexpression had an inhibitory effect on cell migration and invasion. MEG3 overexpression also mediated epithelial-to-mesenchymal transition by significantly enhancing E-cadherin and decreasing $\mathrm{N}$-cadherin, Vimentin and matrix metalloprotein 9 expression levels in NSCLC cells,
\end{abstract}

Correspondence to: Dr Mingli Zhao, Department of Medical Oncology, The Third Affiliated Hospital of Kunming Medical University (Tumor Hospital of Yunnan Province), 519 Kunzhou Road, Xishan, Kunming, Yunnan 650118, P.R. China

E-mail:wuwezi@sohu.com

Abbreviations: MEG3, maternally expressed gene 3; PTEN, phosphatase and tensin homolog; E-cad, E-cadherin; N-cad, $\mathrm{N}$-cadherin; Vim, vimentin; MMP, matrix metalloprotein; RT-qPCR, reverse transcription-quantitative PCR

Key words: non-small cell lung cancer, maternally expressed gene 3, microRNA-21-5p, PTEN, epithelial-to-mesenchymal transition as indicated by western blot analysis. These changes were partially reversed by an miR-21-5p mimic. These results indicated that MEG3 acted as a tumor suppressor that inhibited NSCLC cell migration and invasion via sponging miR-21-5p, which, in turn, enhanced the expression levels of PTEN, in part via the PI3K/AKT signaling pathway. The results of the present study have suggested the potential of MEG3 as a novel therapeutic target for NSCLC treatment.

\section{Introduction}

Lung cancer is the leading cause of cancer-associated mortality worldwide (1). Non-small cell lung cancer (NSCLC) accounts for $\sim 80 \%$ of cases of lung cancer, contributing to the majority of cases in terms of both incidence and mortality (2). Tumor invasion and metastasis are primary reasons for cancer progression and therapy failure (3). Therefore, investigation of the molecular mechanisms underlying NSCLC invasion may be of significance for therapy in patients with metastatic NSCLC.

Long non-coding RNAs (lncRNAs) are a class of non-coding transcripts that are $>200$ nucleotides in length (4). lncRNAs serve essential roles in cell physiological and pathological processes, including proliferation, apoptosis, differentiation, migration and invasion $(5,6)$. A number of dysregulated lncRNAs have been identified in cancer and characterized as oncogenes or tumor suppressors $(7,8)$. For example, maternally expressed gene 3 (MEG3) has been reported to act as an antitumor lncRNA in multiple types of cancer, such as breast, liver, colorectal, lung and gastric cancer (9). Previous studies have suggested that MEG3 is significantly downregulated in NSCLC tissues and cell lines, and that low MEG3 expression levels are associated with poor prognosis $(10,11)$. In vitro experiments have demonstrated that MEG3 overexpression can inhibit cell proliferation, promote apoptosis and enhance chemotherapy sensitivity in NSCLC cells $(11,12)$. However, little is known about the functions and underlying mechanisms of MEG3 in lung cancer metastasis. Given that MEG3 participates in migration and invasion of numerous other types of cancer, including glioma, as well as breast and ovarian cancer (13-15), it was hypothesized that MEG3 may mediate migration and invasion of NSCLC cells. 
As another type of non-coding RNA, microRNAs (miRNAs or miRs) are small (18-22 nucleotides in length) single-stranded transcripts that are able to regulate gene expression levels at the post-transcriptional level by specific binding to the 3'-untranslated region (UTR) of the target mRNA, leading to translational repression or degradation $(16,17)$. Similarly to lncRNAs, miRNAs are also involved in numerous biological behaviors of cancer cells, and aberrant expression levels of miRNAs are an important indicator of cancer $(18,19)$. Previous studies have shown that miR-21-5p is significantly increased in NSCLC cell lines and tissues (20-22); this is positively associated with tumor size, metastasis and poor prognosis of patients with NSCLC $(23,24)$, indicating the oncogenic properties of miR-21-5p. miR-21-5p has been shown to promote NSCLC cell proliferation in vitro $(23,25)$. However, the involvement and mechanisms of miR-21-5p in NSCLC metastasis have yet to be fully elucidated.

Increasing evidence has shown that interactions between lncRNAs and miRNAs have a critical role in potential mechanisms of tumorigenic processes $(26,27)$. IncRNAs can serve as competing endogenous RNAs (ceRNAs) or natural miRNA sponges to modulate miRNA expression levels or sequester miRNAs away from target mRNAs via competitively combining with miRNAs, which decreases mRNA expression levels $(26,27)$. Bioinformatics analysis here suggested putative binding sites for miR-21-5p in MEG3; therefore, it was possible to hypothesize whether MEG3 could function as a ceRNA for miR-21-5p to regulate migration and invasion of NSCLC cells in vitro.

The present study assessed the ability of MEG3 to function as an miR-21-5p sponge to inhibit miR-21-5p expression levels, and, in turn, enhance the expression levels of phosphatase and tensin homolog (PTEN) and suppress the PI3K/AKT signaling pathway in NSCLC PC9 and H1299 cells. Functional analyses demonstrated that MEG3 overexpression could inhibit cell migration, invasion and epithelial-to-mesenchymal transition (EMT). These effects, induced by MEG3, were attenuated by a miR-21-5p mimic. To the best of our knowledge, the present study is the first to identify the molecular mechanism underlying the involvement of the MEG3/miR-21-5p/PTEN axis in NSCLC metastasis.

\section{Materials and methods}

Cell culture. NSCLC cell lines PC9 and H1299 (purchased from Shanghai Cell Bank of Chinese Academy of Sciences) and human lung bronchial epithelial BEAS-2B cells (American Type Culture Collection) were cultured in RPMI-1640 medium supplemented with $10 \%$ fetal bovine serum (both Gibco; Thermo Fisher Scientific, Inc.) and $1 \%(\mathrm{w} / \mathrm{v})$ penicillin/streptomycin (Sigma-Aldrich; Merck KGaA) at $37^{\circ} \mathrm{C}$ in a $5 \% \mathrm{CO}_{2}$ incubator. Cells in the exponential growth phase were used for subsequent experiments.

Target prediction for miR-21-5p. Potential target genes of miR-21-5p were predicted by TargetScan (targetscan.org/), which is a web-based resource used for the prediction of biological targets of miRNAs by searching for the presence of $8 \mathrm{mer}, 7 \mathrm{mer}$ and $6 \mathrm{mer}$ sites that match the seed region of each miRNA.
Reverse transcription-quantitative $(R T-q) P C R$. RNA was extracted from cells using a MagMAX ${ }^{\mathrm{TM}}$ MiRVana $^{\mathrm{TM}}$ Total RNA Isolation kit (Thermo Fisher Scientific, Inc.), and subsequently transcribed into cDNA using a PrimeScript ${ }^{\mathrm{TM}}$ RT reagent kit (Takara Biotechnology Co., Ltd.) according to the manufacturers' protocols. RT-qPCR was performed using SYBR Green PCR Master Mix on an ABI 7300 Real-Time PCR System (both Applied Biosystems; Thermo Fisher Scientific, Inc.) with customized primer sets for MEG3, PTEN and miR-21-5p. RT-qPCR amplification included a holding stage at $95^{\circ} \mathrm{C}$ for $10 \mathrm{~min}$, followed by 40 cycles of $95^{\circ} \mathrm{C}$ for $15 \mathrm{sec}$ and $60^{\circ} \mathrm{C}$ for $1 \mathrm{~min}$. The subsequent melt curve stage consisted of $95^{\circ} \mathrm{C}$ for $15 \mathrm{sec}, 60^{\circ} \mathrm{C}{ }^{\circ}$ for $1 \mathrm{~min}$ and $95^{\circ} \mathrm{C}$ for 15 sec. GAPDH was used as the internal control for MEG3 and PTEN, whereas U6 was used as the internal control for miR-21-5p. The relative fold change of gene expression levels was determined using the $2^{-\Delta \Delta \mathrm{Cq}}$ method (28). Primers (synthesized by Sangon Biotech Co., Ltd.) are listed in Table I.

Cell transfection. The pcDNA3.1-MEG3 plasmid (termed MEG3) was constructed by sub-cloning MEG3 sequence into pcDNA 3.1 vector (Shanghai GenePharma Co., Ltd.). The cells were transfected with MEG3 and and pcDNA 3.1 empty vector (vector) to a final concentration of $100 \mathrm{nM}$ using Lipofectamine ${ }^{\circledR} 2000$ (cat. no. 11668019; Invitrogen; Thermo Fisher Scientific, Inc.) according to the manufacturer's protocols. miR-21-5p inhibitor (5'-UAGCUUAUCAGACUG AUGUUGA-3'), miR-21-5p mimic (5'-UAGCUUAUCAGA CUGAUGUUGA-3') and their negative controls (NC, 5'-CCC AGAATGTTGACAGCTGCCTCTT-3') were synthesized by Guangzhou RiboBio Co., Ltd. The cells were transfected with miR-21-5p mimic or inhibitor to a final concentration of $50 \mathrm{nM}$ using Lipofectamine 2000 according to the manufacturer's protocols. Subsequent experiments were performed $24 \mathrm{~h}$ after transfection.

In order to assess the effects of miR-21-5p on MEG3-mediated cellular responses, cells were transfected with MEG3, miR-21-5p mimic, miR-21-5p inhibitor, MEG3+miR-21-5p mimic and their negative controls using Lipofectamine 2000, respectively.

Luciferase reporter assay. The 3'-UTR regions of MEG3 or PTEN containing the predicted miR-21-5p specific binding sites were amplified by PCR and cloned into the firefly luciferase reporter vector, pmirGLO (Promega Corporation), to obtain the wild-type luciferase reporter plasmids, wt-MEG3 and wt-PTEN, respectively. In order to generate the mutant reporter plasmids mut-MEG3 and mut-PTEN, certain nucleotides in MEG3 or PTEN 3'-UTR were mutated using PCR lacking miR-21-5p-binding sites. The constructed luciferase reporter plasmids (wt-MEG3, mut-MEG3, wt-PTEN and mut-PTEN) were separately co-transfected with miR-21-5p mimic or mimic control into PC9 and H1299 cells using Lipofectamine 2000. Cells were lysed and luciferase activity was assayed at $24 \mathrm{~h}$ post-transfection using a Dual-Luciferase Reporter Assay system (Promega Corporation), according to the manufacturer's instructions.

Western blotting. Proteins were extracted from cells using ice-cold RIPA lysis buffer (Beyotime Institute of 
Table I. Primers for reverse transcription-quantitative PCR.

\begin{tabular}{lll}
\hline Name & Direction & Sequence (5'-3') \\
\hline Maternally expressed gene 3 & Forward & TCCATGCTGAGCTGCTGCCAAG \\
& Reverse & AGTCGACAAAGACTGACACCC \\
PTEN & Forward & CCCAGTCAGAGGCGCTATG \\
& Reverse & GGCAGACCACAAACTGAGGATT \\
GAPDH & Forward & AATGGACAACTGGTCGTGGAC \\
MicroRNA-21-5p & Reverse & CCCTCCAGGGGATCTGTTTG \\
& Forward & GCACCTAGCTTATCAGACTGA \\
U6 & Reverse & GTGCAGGGTCCGAGGT \\
& Forward & GCTTCGGCAGCATATACTAAAAT \\
& Reverse & CGCTTCACGAATTTGCGTGTCAT \\
\hline
\end{tabular}

PTEN, phosphatase and tensin homolog.

Biotechnology). Following quantification using a BCA kit (Thermo Fisher Scientific, Inc.), $40 \mu \mathrm{g}$ total protein of each sample was denatured in a boiling water bath and separated by SDS-PAGE (10\% gels), then transferred to a polyvinylidene fluoride membrane. Subsequently, the membranes were probed with 5\% non-fat milk in TBS at room temperature for $1 \mathrm{~h}$, and incubated with specific primary antibodies for PTEN (1:500; cat. no. ab31392; Abcam), N-cadherin (N-cad; 1:500; cat. no. ab76011; Abcam), E-cadherin (E-cad; 1:500; cat. no. ab1416; Abcam), Vimentin (Vim; 1:500; cat. no. ab137321; Abcam), matrix metalloprotein (MMP)9 (1:500; cat no. ab38898; Abcam), PI3K (1:500; cat. no. GW21071; Sigma-Aldrich; Merck KGaA), phosphorylated (p)-PI3K (1:1,000; cat. no. 4228; Cell Signaling Technology, Inc.), AKT (1:1,000; cat. no. SAB4500797; Sigma-Aldrich; Merck KGaA), p-AKT (1:1,000, cat. no. 9271; Cell Signaling Technology, Inc.) and $\beta$-actin (1:1,000; cat. no. 3700; Cell Signaling Technology, Inc.) at $4^{\circ} \mathrm{C}$ overnight. After being washed five times, the membranes were incubated with horseradish peroxidase-conjugated goat anti-rabbit IgG (1:2,000; cat. no. ab6721; Abcam) at room temperature for $2 \mathrm{~h}$, and detected using a Novex ${ }^{\circledR}$ ECL Chemiluminescent Substrate Reagent kit (Thermo Fisher Scientific, Inc.) and ChemiDoc ${ }^{\mathrm{TM}} \mathrm{XRS}^{+}$imaging system (Bio-Rad Laboratories, Inc.). The signal intensity was quantified using ImageJ software (version 5.0; National Institutes of Health).

Migration and invasion assays. Cell migration was assessed using 24-well Transwell filters (Corning Life Sciences). Briefly, a total of $5 \times 10^{4}$ cells was seeded into the upper chamber in serum-free medium, while the lower chamber was filled with complete medium containing $20 \%$ FBS. After $24 \mathrm{~h}$ incubation at $37^{\circ} \mathrm{C}$, cells that passed through the filter were fixed using $5 \%$ glutaraldehyde at $4^{\circ} \mathrm{C}$ for $30 \mathrm{~min}$ and stained with $0.5 \%$ crystal violet solution at $37^{\circ} \mathrm{C}$ for $30 \mathrm{~min}$. Images were captured using an inverted light microscope (magnification, x200; Olympus Corporation) and cells in five randomly selected fields were counted. For cell invasion assay, the filter membranes were pre-coated with Matrigel ${ }^{\mathrm{TM}}$
(Beijing Solarbio Science \& Technology Co., Ltd.) at $37^{\circ} \mathrm{C}$ for $30 \mathrm{~min}$, and other steps were performed as described in the migration assay.

Statistical analysis. All data were analyzed using GraphPad Prism software (version 5; GraphPad Software, Inc.) and are expressed as the mean \pm SD of three independent repeats. Significant differences were analyzed by one-way ANOVA to compare $>2$ groups, followed by Tukey's post hoc test. $\mathrm{P}<0.05$ was considered to indicate a statistically significant difference.

\section{Results}

MEG3 suppresses miR-21-5p expression levels and acts as a sponge of $m i R-21-5 p$. First, RT-qPCR was used to analyze the expression levels of MEG3 in NSCLC cell lines. The results demonstrated that MEG3 was downregulated in the NSCLC cell lines PC9 and H1299 compared with normal lung bronchial epithelial BEAS-2B cells (Fig. 1A). Next, to investigate the effect of MEG3 on miR-21-5p expression levels, MEG3 was overexpressed by transfecting MEG3-overexpressing plasmid in PC9 and H1299 cells. The expression levels of MEG3 were shown to be upregulated, assessed via RT-qPCR (Fig. 1B). MEG3 overexpression significantly suppressed the expression levels of miR-21-5p (Fig. 1C).

In order to investigate whether MEG3 functions as a sponge of miR-21-5p to regulate its expression levels, the online database TargetScan (targetscan.org) was used to predict the association between MEG3 and miR-21-5p. Bioinformatics analysis demonstrated that miR-21-5p directly interacts with MEG3 via sequence complementarity (Fig. 1D). In order to verify the direct binding of miR-21-5p to MEG3, luciferase reporter plasmids containing wt-MEG3 or mut-MEG3 were co-transfected with either miR-21-5p mimic or NC-mimic into cells. As expected, miR-21-5p mimic significantly repressed the luciferase intensity of wt-MEG3, but had no effect on the luciferase activity of mut-MEG3 (Fig. 1E). These results demonstrated that MEG3 repressed miR-21-5p expression levels via directly targeting miR-21-5p. 

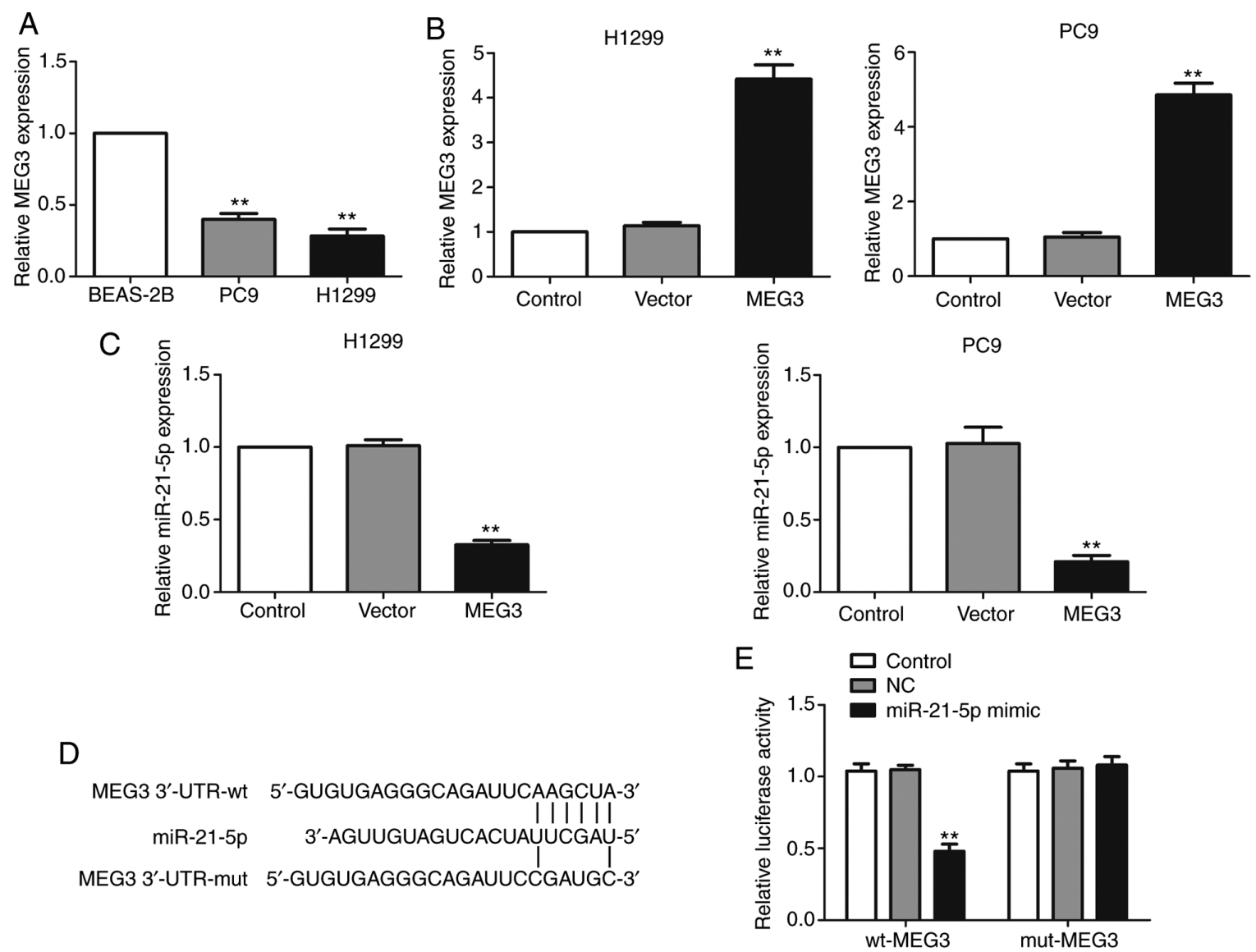

Figure 1. MEG3 acts as a molecular sponge to suppress miR-21-5p expression levels. RT-qPCR analysis of MEG3 expression levels in (A) non-transfected and (B) MEG3-transfected non-small cell lung cancer cell lines PC9 and H1299. (C) RT-qPCR analysis of miR-21-5p expression levels in MEG3-transfected PC9 and H1299 cells. (D) Sequence alignment between miR-21-5p and MEG3. The wt binding sites of miR-21-5p on MEG3 and its mutated binding sequences are shown. (E) Luciferase activity in cells following co-transfection with miR-21-5p mimic and luciferase reporters containing wt- or mut-MEG3 transcript. Data are presented as the mean $\pm \mathrm{SD}$. ${ }^{* *} \mathrm{P}<0.01$ vs. control. MEG3, maternally expressed gene 3; miR, microRNA; RT-q, reverse transcription-quantitative; wt, wild-type; mut, mutant; UTR, untranslated region; NC, negative control.

PTEN is a direct target of miR-21-5p. First, the expression levels of miR-21-5p in NSCLC cell lines were evaluated via RT-qPCR; the results showed that miR-21-5p was substantially increased in the two NSCLC cell lines PC9 and H1299 in comparison with BEAS-2B cells (Fig. 2A). PTEN was predicted to be a candidate target gene of miR-21-5p using TargetScan (targetscan.org) (Fig. 2E). Subsequently, cells were transfected with miR-21-5p inhibitor to determine whether the expression levels of PTEN were regulated by miR-21-5p. Suppressed expression levels of miR-21-5p were confirmed via RT-qPCR (Fig. 2B), and significant increases in PTEN expression, at both the mRNA (Fig. 2C) and protein (Fig. 2D) levels, were observed following miR-21-5p inhibitor transfection.

Next, it was determined whether the enhanced PTEN expression levels were caused by the direct binding of miR-21-5p to the 3'-UTR of PTEN. To this end, PTEN 3'-UTR containing the wild-type or mutated miR-21-5p target site was cloned in a firefly luciferase reporter vector to obtain the reporter plasmids wt-PTEN and mut-PTEN, respectively. Following miR-21-5p mimic and wt-PTEN co-transfection, the luciferase activity was markedly attenuated, whereas co-transfection with miR-21-5p and mut-PTEN had little effect on luciferase activity (Fig. 2F). These data indicated that miR-21-5p inhibited PTEN expression levels by directly binding to PTEN 3'-UTR.

MEG3 positively regulates PTEN expression levels by sponging $m i R-21-5 p$. It was further investigated whether MEG3 targeted PTEN expression levels by sponging miR-21-5p. miR-21-5p mimic and MEG3-overexpressing plasmid were co-transfected into H1299 cells, and PTEN expression at both the mRNA (Fig. 3A) and protein (Fig. 3B) levels was analyzed. The results indicated that upregulation of miR-21-5p significantly repressed MEG3-induced increased expression levels of PTEN.

MEG3 mediates the EMT process via sponging miR-21-5p. Given the role of EMT in cancer migration and invasion, it was investigated whether MEG3 or miR-21-5p mediated EMT. Transfection with MEG3-overexpressing plasmid or miR-21-5p inhibitor significantly increased levels of the epithelial marker E-cad, but decreased levels of the mesenchymal markers N-cad, Vim and MMP9 (Fig. 4A and C). Then, to determine whether MEG3 mediated the EMT process via sponging miR-21-5p, mir-21-5p mimic or NC-mimic was co-transfected with MEG3-overexpressing plasmid in PC9 and H1299 cells. The alterations in expression 
A
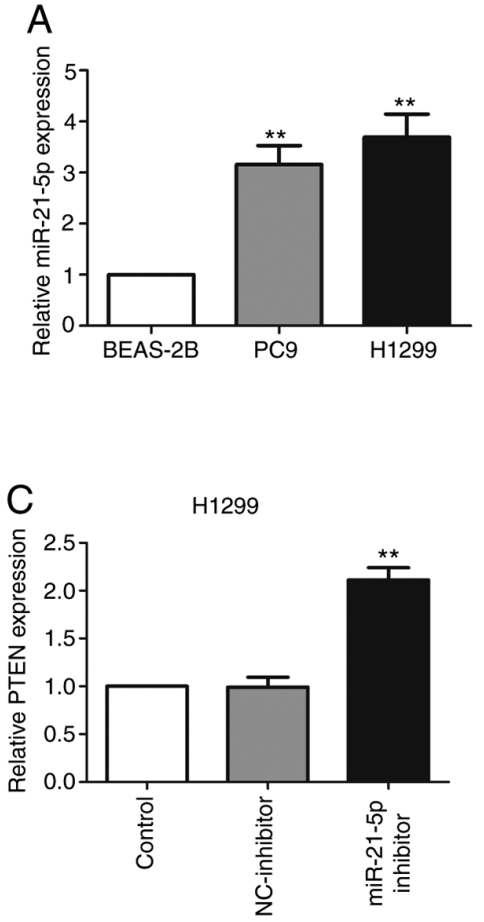

H1299

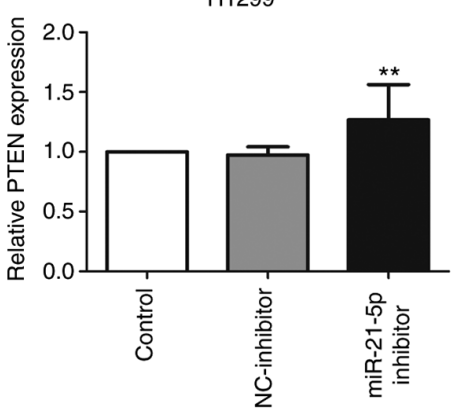

E
$B$ 。

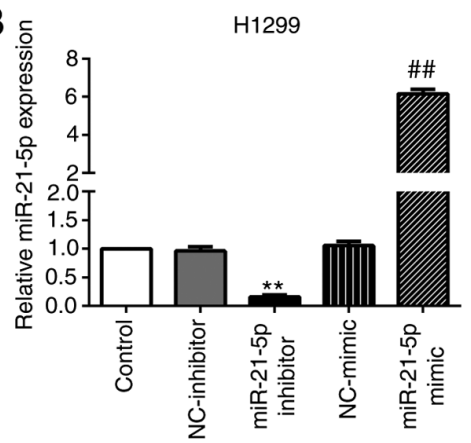

PC9
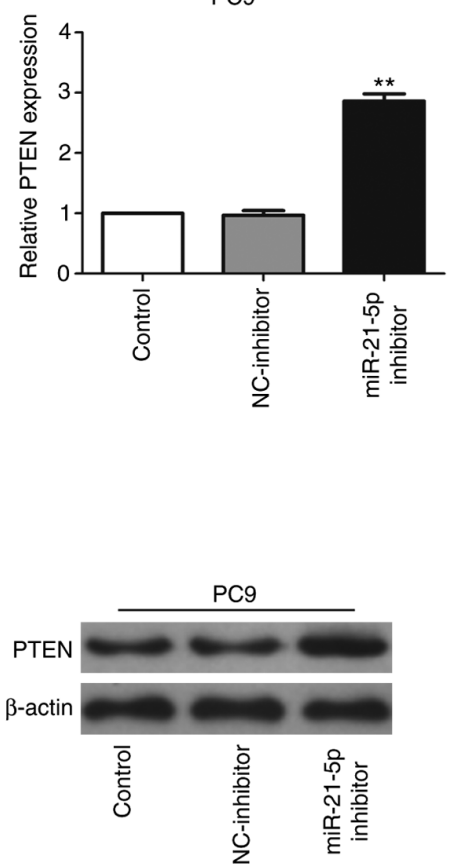

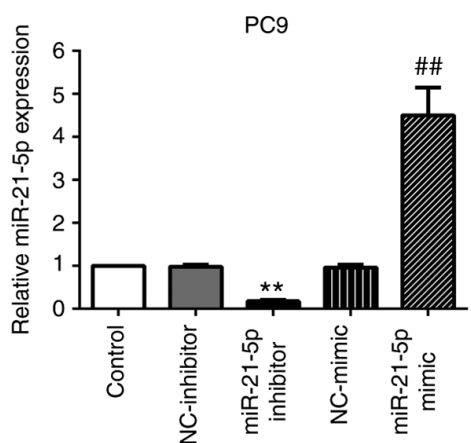

D

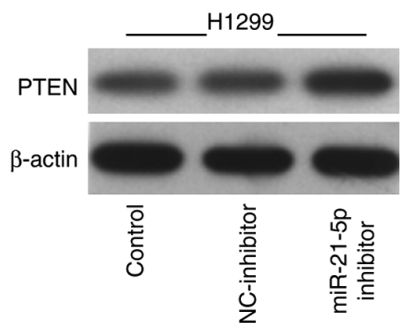

PC9

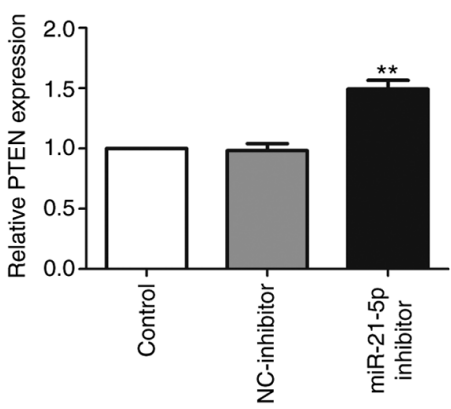

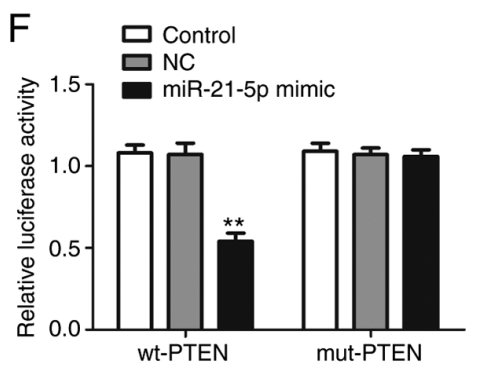

Figure 2. miR-21-5p targets PTEN to suppress its expression levels. RT-qPCR analysis of miR-21-5p expression levels in (A) non-transfected cells, and (B) the miR-21-5p inhibitor and miR-21-5p mimic-transfected non-small cell lung cancer cell lines, PC9 and H1299. (C) RT-qPCR analysis and (D) representative western blotting of PTEN expression levels in miR-21-5p inhibitor-transfected PC9 and H1299 cells. (E) Sequence alignment between miR-21-5p and PTEN. The wt binding sites of miR-21-5p on PTEN and its mutated binding sequences are shown. (F) Luciferase activity in cells following co-transfection with miR-21-5p mimic and luciferase reporters containing wt- or mut-PTEN transcript. Data are presented as the mean $\pm \mathrm{SD}$. ${ }^{* *} \mathrm{P}<0.01 \mathrm{vs}$. control; ${ }^{\#} \mathrm{P}<0.01 \mathrm{vs}$. NC-mimic. miR, microRNA; RT-q, reverse transcription-quantitative; wt, wild-type; mut, mutant; NC, negative control; UTR, untranslated region; PTEN, phosphatase and tensin homolog.

levels of epithelial and mesenchymal markers induced by MEG3 were significantly abrogated by miR-21-5p mimic (Fig. 4A and C).

MEG3/miR-21-5p/PTEN axis function may be mediated via the PI3K/AKT signaling pathway. PTEN has been reported to be an antagonist regulator of the PI3K/AKT pathway, which is involved in the regulation of cancer migration and invasion (29).
It was therefore speculated that the MEG3/miR-21-5p/PTEN axis may function via the PI3K/AKT signaling pathway. In order to investigate this, phosphorylation of PI3K and AKT in cells transfected with MEG3-overexpressing plasmid was analyzed. MEG3 overexpression significantly decreased phosphorylation of both PI3K and AKT (Fig. 4B and D). Similar patterns of p-PI3K and p-AKT were observed in cells transfected with miR-21-5p inhibitor (Fig. 4B and D). The participation of 


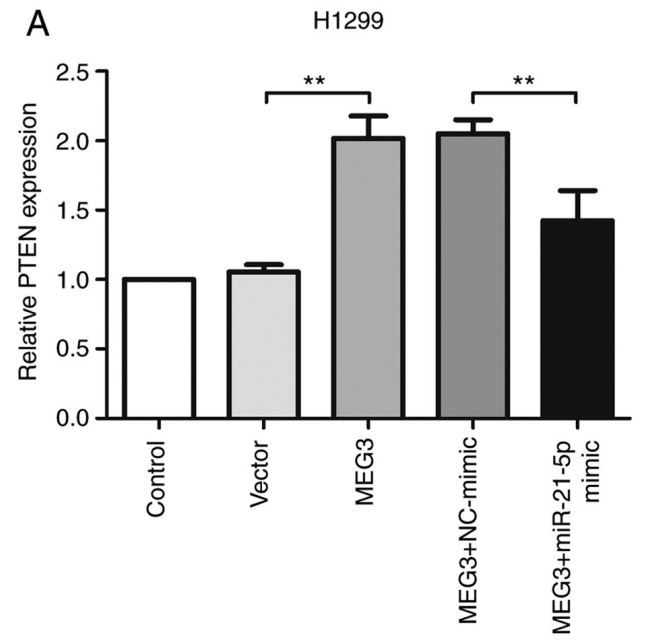

B
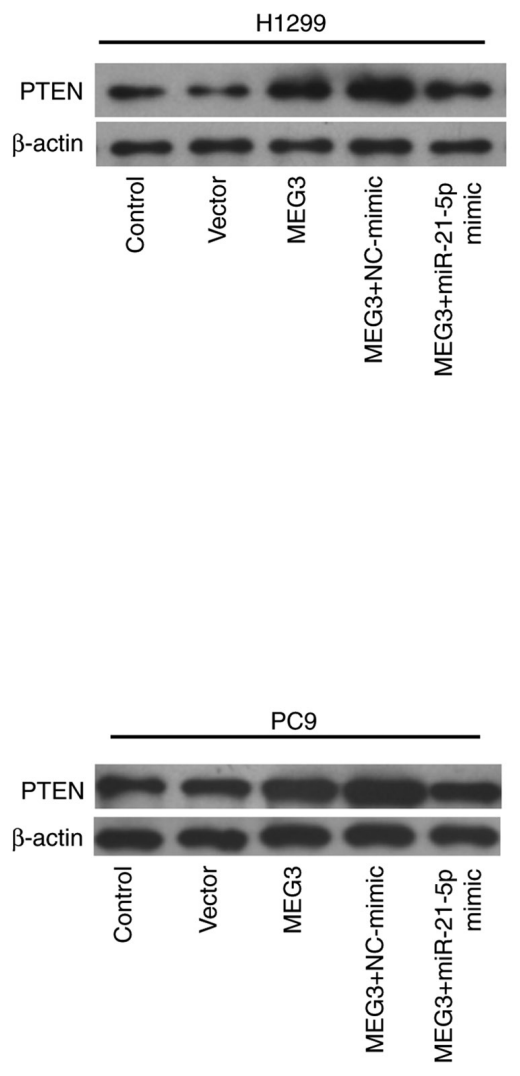

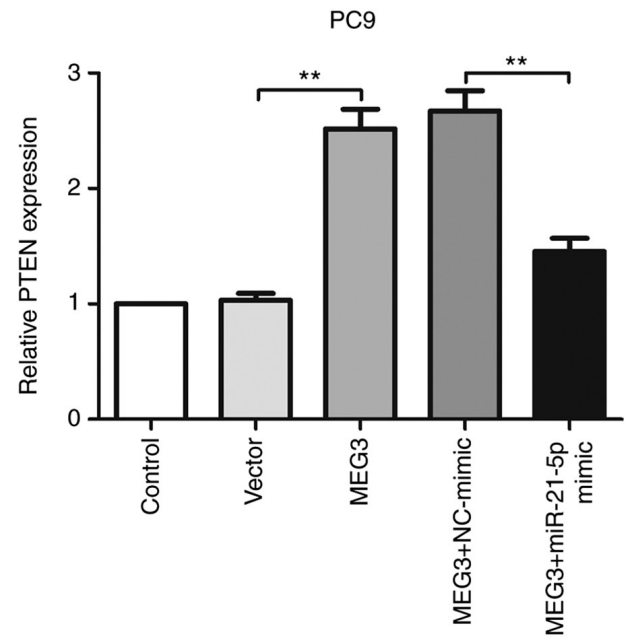

H1299

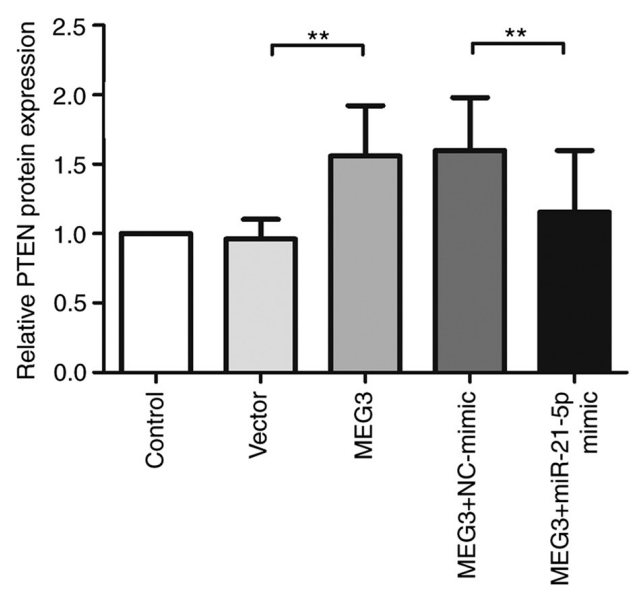

PC9

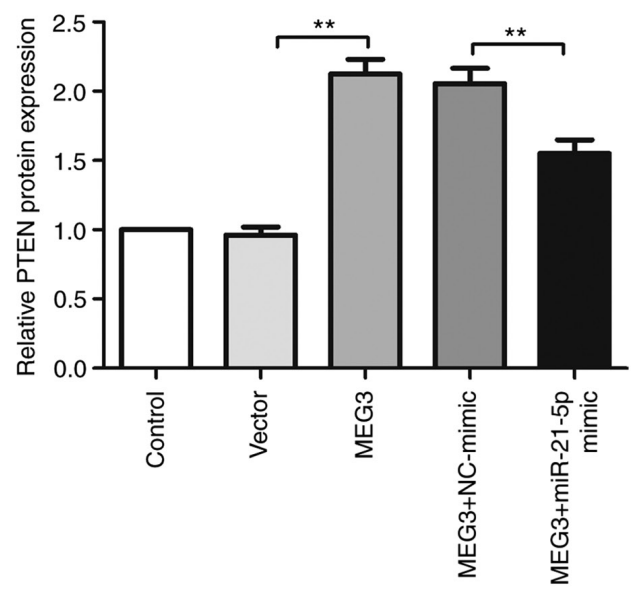

Figure 3.MEG3 enhances PTEN expression levels via modulating miR-21-5p. PC9 and H1299 cells were transfected with MEG3, empty vector,MEG3+ miR-21-5p mimic and MEG3+ NC-mimic. (A) Reverse transcription-quantitative PCR analysis of PTEN mRNA expression levels in cells. (B) Representative western blots, and the corresponding semi-quantitative analysis of relative changes in expression levels of PTEN protein in cells. ** P $<0.01$. MEG3, maternally expressed gene 3; miR, microRNA; NC, negative control; PTEN, phosphatase and tensin homolog.

miR-21-5p in MEG3-mediated regulation of the PI3K/AKT pathway was further investigated by co-transfecting miR-21-5p mimic or NC-mimic with MEG3-overexpressing plasmid into PC9 and H1299 cells. The results showed that the decreases in phosphorylation levels of PI3K/AKT induced by MEG3 were significantly abrogated by miR-21-5p (Fig. 4B and D).
MEG3 attenuates cell migration and invasion via sponging $m i R-21-5 p$. The effect of MEG3 on cell migration and invasion was evaluated by transfecting MEG3-overexpressing or empty vector plasmid into PC9 and H1299 cells. Transwell migration and invasion assays showed that MEG3 overexpression induced a significant decrease in the number 

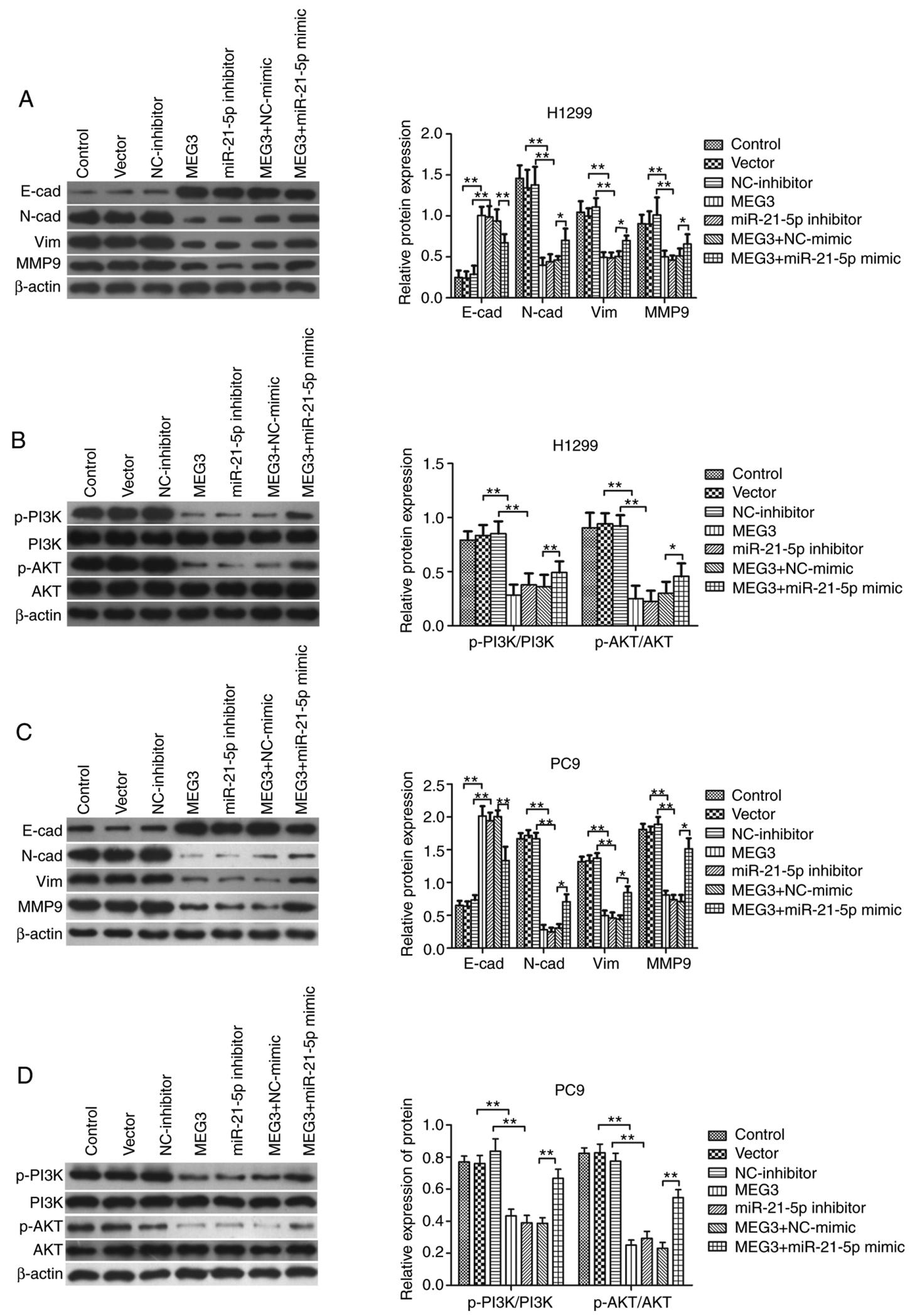

Figure 4. MEG3 suppresses the PI3K/AKT pathway and the epithelial-to-mesenchymal transition process via miR21-5p. PC9 and H1299 cells were transfected with MEG3, empty vector, miR-21-5p inhibitor, NC-inhibitor, MEG3+ miR-21-5p mimic and MEG3+ NC-mimic. Representative western blots, and semi-quantitative analysis of relative changes in expression levels of E-cad, N-cad, Vim and MMP9 in (A) H1299 and (C) PC9 cells. The protein expression levels of p-PI3K, PI3K, p-AKT and AKT protein in (B) H1299 and (D) PC9 cells are also shown. ${ }^{* *} \mathrm{P}<0.01 .{ }^{*} \mathrm{P}<0.05$. MEG3, maternally expressed gene 3; miR, microRNA; NC, negative control; E-cad, E-cadherin; N-cad, N-cadherin; Vim, vimentin; MMP, matrix metalloprotein; p-, phosphorylated.

of the migratory and invasive cells compared with empty vector control (Fig. 5A-D). Similar results were observed in cells transfected with miR-21-5p inhibitor; miR-21-5p attenuation significantly decreased migration and invasion of PC9 and H1299 cells compared with NC-inhibitor (Fig. 5A-D). 

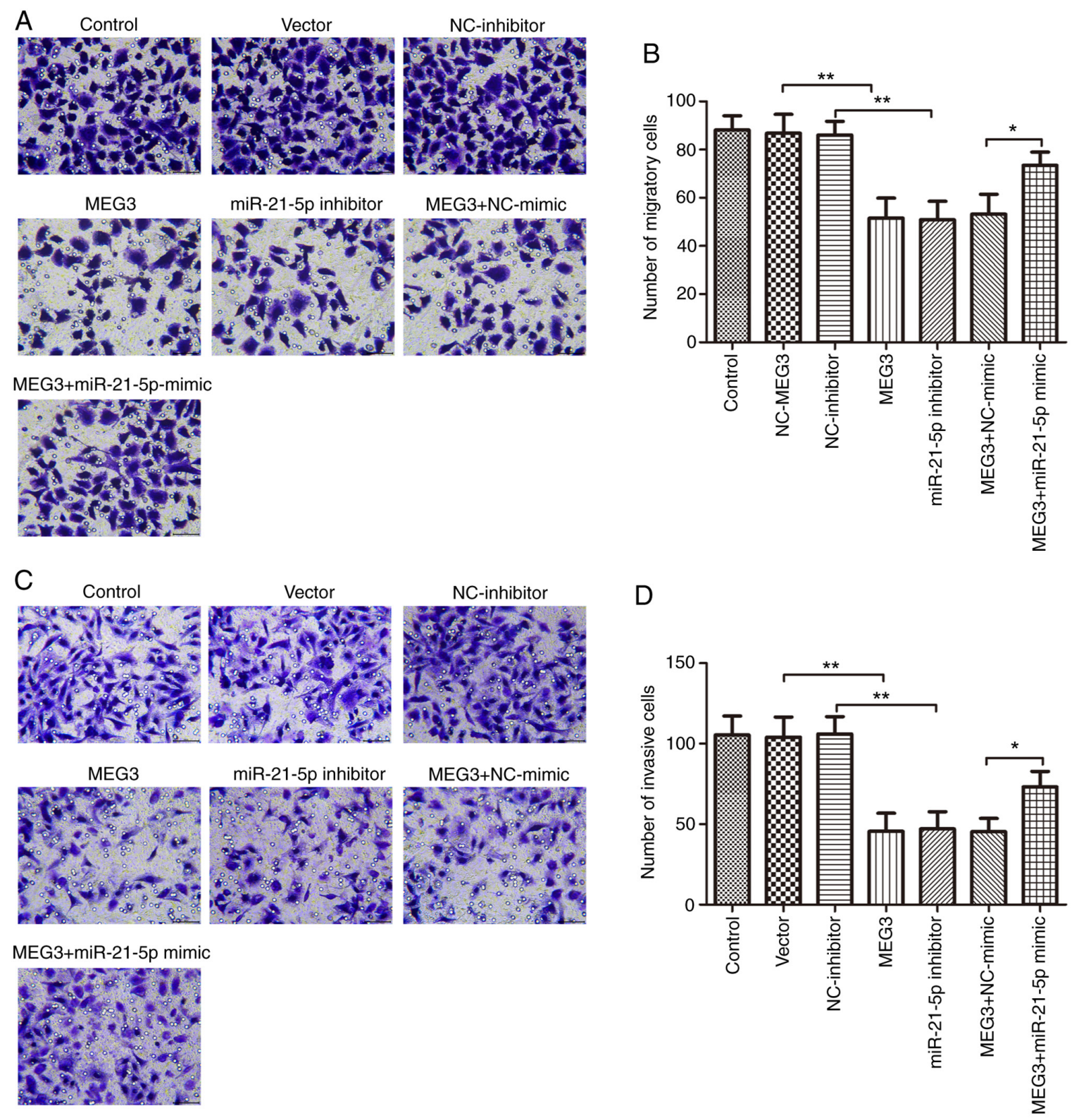

Figure 5. MEG3 suppresses migration and invasion via miR-21-5p. PC9 and H1299 cells were transfected with MEG3, empty vector, miR-21-5p inhibitor, NC-inhibitor, MEG3+ miR-21-5p mimic and MEG3 + NC-mimic. (A) Transwell migration assay. (B) Quantitative analysis of migratory cells passing through the membrane. (C) Transwell invasion assay. (D) Quantitative analysis of invasive cells passing through the membrane. ${ }^{* *} \mathrm{P}<0.01$. "P<0.05. MEG3, maternally expressed gene 3; miR, microRNA; NC, negative control.

The contribution of miR-21-5p to the effect of MEG3 on biological behaviors of PC9 and H1299 cells was next investigated. miR-21-5p mimic or NC-mimic was co-transfected with MEG3-overexpressing plasmid into PC9 and H1299 cells, and the migration and invasion abilities were assessed. The results demonstrated that miR-21-5p mimic significantly mitigated the inhibitory effects of MEG3 on cell migration and invasion (Fig. 5A-D). These results indicated that MEG3 involvement in NSCLC cell migration and invasion was mediated, at least partially, by sponging and suppressing miR-21-5p.

\section{Discussion}

lncRNAs have been implicated in cancer development $(7,8)$. MEG3 has been reported to function as a tumor suppressor in a number of types of cancer (9), such as lung (10), breast (13) and ovarian cancer (15). Numerous studies have identified low expression levels of MEG3 in NSCLC tissues and cell lines $(10,11)$; similarly, the present study demonstrated that MEG3 was downregulated in NSCLC PC9 and H1299 cells. Previous studies have also suggested that MEG3 overexpression may inhibit proliferation and induce apoptosis in NSCLC cells $(10,11)$. In the present study, MEG3 overexpression suppressed the migration and invasion abilities of NSCLC PC9 and H1299 cells in vitro. EMT is a key process associated with cancer cell migration and invasion. The present results showed that MEG3 overexpression inhibited the EMT process by upregulating the expression levels of the epithelial marker, E-cad, and downregulating the expression levels of the mesenchymal markers, N-cad, Vim and MMP9, in PC9 and H1299 cells. MEG3 has been previously reported to show similar 
effects on the migration and invasion abilities of pancreatic cancer cells in vitro (30).

lncRNAs regulate cancer development via sponging miRNAs $(26,27)$. MEG3 functions via sponging multiple miRNAs in numerous types of cancer $(31,32)$. In NSCLC, for instance, MEG3 has been reported to act as a molecular sponge of miR-7-5p and miR-3163 to inhibit cell growth (10,33). miR-21-5p has been reported to be an oncogene in NSCLC (20-24). The present study showed a strong association between miR-21-5p and MEG3 in PC9 and H1299 cells. MEG3 overexpression significantly inhibited miR-21-5p expression levels in PC9 and H1299 cells, and dual luciferase assays demonstrated that MEG3 directly interacted with miR-21-5p. These results are in agreement with a previous report by Wang et al (34). Furthermore, an miR-21-5p mimic significantly restored the changes induced by MEG3 on cell migration, invasion and the EMT process. Additionally, miR-21-5p attenuation also suppressed cell migration, invasion and the EMT process in PC9 and H1299 cells. These results suggested that MEG3 inhibited migration and invasion of H1299 cells via acting as a miR-21-5p sponge. Previous studies have also indicated the involvement of the MEG3/miR-21-5p axis in cell proliferation and apoptosis in NSCLC and cervical cancer $(34,35)$.

PTEN has proved to be a powerful tumor suppressor, and low levels of PTEN are one of the most frequent events observed in a variety of types of cancer $(36,37)$. Numerous studies have observed decreased expression levels of PTEN in NSCLC tissues and cell lines $(29,38)$, and PTEN overexpression has been reported to inhibit the migration and invasion of NSCLC cells (39). Notably, evidence suggests that PTEN expression levels may be downregulated by miRNAs involved in cancer development, including those involved in NSCLC $(29,40)$. In the present study, dual luciferase assay demonstrated that PTEN was a direct target of miR-21-5p in H1299 lung cancer cells, and attenuation of miR-21-5p enhanced PTEN expression levels. The miR-21-5p/PTEN axis has also been reported in other types of cancer, such as breast and epithelial ovarian cancer $(41,42)$. Furthermore, in the present study, MEG3 overexpression resulted in increased PTEN expression levels, which was mitigated by an miR-21-5p mimic in PC9 and H1299 cells. In addition, PTEN has been reported to function by negatively regulating the PI3K/AKT signaling pathway, which is activated in NSCLC development (38). As expected, MEG3 overexpression also caused suppression of the PI3K/AKT signaling pathway, whereas these effects were reversed by miR-21-5p in PC9 and H1299 cells. These data suggested that MEG3 upregulated PTEN expression levels and subsequently attenuated the PI3K/AKT signaling pathway, partially by sponging miR-21-5p, and thus inhibited the migration and invasion of NSCLC PC9 and H1299 cells.

Taken together, the results of the present study have demonstrated that MEG3 inhibited migration and invasion of NSCLC PC9 and H1299 cells, partially by regulating the miR-21-5p/PTEN axis and the PI3K/AKT signaling pathway. Therefore, the MEG3/miR-21-5p/PTEN axis may represent a novel target pathway for NSCLC therapy.

\section{Acknowledgements}

Not applicable.

\section{Funding}

No funding was received.

\section{Availability of data and materials}

The datasets used and/or analyzed during the current study are available from the corresponding author on reasonable request.

\section{Authors' contributions}

DL, YL and MZ performed the experiments, and collected and interpreted data. YL, QB and JD participated in the design and coordination of experimental work, and acquisition of data. DL and NW participated in the study design, data collection, analysis of data and preparation of the manuscript. $\mathrm{SH}$ designed the study, analyzed and interpreted data and drafted the manuscript. All authors read and approved the final manuscript.

\section{Ethics approval and consent to participate}

Not applicable.

\section{Patient consent for publication}

Not applicable.

\section{Competing interests}

The authors declare that they have no competing interests.

\section{References}

1. Torre LA, Siegel RL and Jemal A: Lung cancer statistics. Adv Exp Med Biol 893: 1-19, 2016.

2. Zarogoulidis K, Zarogoulidis P, Darwiche K, Boutsikou E, Machairiotis N, Tsakiridis K, Katsikogiannis N, Kougioumtzi I, Karapantzos I, Huang H, et al: Treatment of non-small cell lung cancer (NSCLC). J Thorac Dis 5 (Suppl 4): S389-396, 2013.

3. Domoto T, Pyko IV, Furuta T, Miyashita K, Uehara M, Shimasaki T and Nakada M, Minamoto T: Glycogen synthase kinase- $3 \beta$ is a pivotal mediator of cancer invasion and resistance to therapy. Cancer Sci 107: 1363-1372, 2016.

4. Jeong S, Lee J, Kim D, Seol MY, Lee WK, Jeong JJ, Nam KH, Jung SG, Shin DY, Lee EJ, et al: Relationship of focally amplified long noncoding on chromosome 1 (FAL1) lncRNA with E2F transcription factors in thyroid cancer. Medicine (Baltimore) 95: e2592, 2016.

5. Lian Y, Xiao C, Yan C, Chen D, Huang Q, Fan Y, Li Z and Xu H: Knockdown of pseudogene derived from lncRNA DUXAP10 inhibits cell proliferation, migration, invasion, and promotes apoptosis in pancreatic cancer. J Cell Biochem 119: 3671-3682, 2018.

6. Dong X, Chen R, Lin H, Lin T and Pan S: IncRNA BG981369 inhibits cell proliferation, migration, and invasion, and promotes cell apoptosis by SRY-related high-mobility group box 4 (SOX4) signaling pathway in human gastric Cancer. Med Sci Monit 24: 718-726, 2018.

7. Ponzio G, Rezzonico R, Bourget I, Allan R, Nottet N, Popa A, Magnone V, Rios G, Mari B and Barbry P: A new long noncoding RNA (lncRNA) is induced in cutaneous squamous cell carcinoma and down-regulates several anticancer and cell differentiation genes in mouse. J Biol Chem 292: 12483-12495, 2017.

8. Zhao JH, Sun JX, Song YX, Chen XW, Yang YC, Ma B, Wang J, Gao P and Wang ZN: A novel long noncoding RNA-LOWEG is low expressed in gastric cancer and acts as a tumor suppressor by inhibiting cell invasion. J Cancer Res Clin Oncol 142: 601-609, 2016. 
9. Al-Rugeebah A, Alanazi M and Parine NR: MEG3: An oncogenic long non-coding RNA in different cancers. Pathol Oncol Res 25: 859-874, 2019.

10. Wu JL, Meng FM and Li HJ: High expression of 1ncRNA MEG3 participates in non-small cell lung cancer by regulating microRNA-7-5p. Eur Rev Med Pharmacol Sci 22: 5938-5945, 2018.

11. Lu KH, Li W, Liu XH, Sun M, Zhang ML, Wu WQ, Xie WP and Hou YY: Long non-coding RNA MEG3 inhibits NSCLC cells proliferation and induces apoptosis by affecting p53 expression. BMC Cancer 13: 461, 2013.

12. Xia Y, He Z, Liu B, Wang P and Chen Y: Downregulation of Meg3 enhances cisplatin resistance of lung cancer cells through activation of the WNT/ $\beta$-catenin signaling pathway. Mol Med Rep 12: 4530-4537, 2015.

13. Sun L, Li Y and Yang B: Downregulated long non-coding RNA MEG3 in breast cancer regulates proliferation, migration and invasion by depending on $\mathrm{p} 53$ 's transcriptional activity. Biochem Biophys Res Commun 478: 323-329, 2016.

14. Qin N, Tong GF, Sun LW and Xu XL: Long Noncoding RNA MEG3 Suppresses Glioma Cell Proliferation, Migration, and Invasion by Acting as a Competing Endogenous RNA of miR-19a. Oncol Res 25: 1471-1478, 2017.

15. Wang J, Xu W, He Y, Xia Q and Liu S: lncRNA MEG3 impacts proliferation, invasion, and migration of ovarian cancer cells through regulating PTEN. Inflamm Res 67: 927-936, 2018.

16. Hujie G, Zhou SH, Zhang H, Qu J, Xiong XW, Hujie O, Liao CG and Yang SE: MicroRNA-10b regulates epithelial-mesenchymal transition by modulating KLF4/KLF11/Smads in hepatocellular carcinoma. Cancer Cell Int 18: 10, 2018.

17. Li C, Jiang Y, Miao R, Qu K, Zhang J and Liu C: MicroRNA-1271 functions as a metastasis and epithelial-mesenchymal transition inhibitor in human HCC by targeting the PTP4A1/c-Src axis. Int J Oncol 52: 536-546, 2018.

18. Lages E, Ipas H, Guttin A, Nesr H, Berger F and Issartel JP: MicroRNAs: Molecular features and role in cancer. Front Biosci (Landmark Ed) 17: 2508-2540, 2012.

19. Calin GA and Croce CM: MicroRNA signatures in human cancers. Nat Rev Cancer 6: 857-866, 2006.

20. Yang C, Sun C, Liang X, Xie S, Huang J and Li D: Integrative analysis of microRNA and mRNA expression profiles in non-small-cell lung cancer. Cancer Gene Ther 23: 90-97, 2016.

21. Tian F, Li R, Chen Z, Shen Y,Lu J, Xie X and Ge Q: Differentially Expressed miRNAs in tumor, adjacent, and normal tissues of lung adenocarcinoma. Biomed Res Int 2016: 1428271, 2016.

22. Wang K, Chen $\mathrm{M}$ and $\mathrm{Wu} \mathrm{W}$ : Analysis of microRNA (miRNA) expression profiles reveals 11 key biomarkers associated with non-small cell lung cancer. World J Surg Oncol 15: 175, 2017.

23. Li $X$ and $\mathrm{Wu} X$ : MiR-21-5p promotes the progression of non-small-cell lung cancer by regulating the expression of SMAD7. Onco Targets Ther 11: 8445-8454, 2018.

24. Li C, Yin Y, Liu X, Xi X, Xue W and Qu Y: Non-small cell lung cancer associated microRNA expression signature: Integrated bioinformatics analysis, validation and clinical significance. Oncotarget 8: 24564-24578, 2017.

25. Yan L, Ma J, Wang Y, Zan J, Wang Z, Zhu Y, Zhu Y, Ling L, Cao L, Liu X, et al: miR-21-5p induces cell proliferation by targeting TGFBI in non-small cell lung cancer cells. Exp Ther Med 16: 4655-4663, 2018.

26. Liz J and Esteller M: lncRNAs and microRNAs with a role in cancer development. Biochim Biophys Acta 1859: 169-176, 2016.

27. Tay Y, Rinn J and Pandolfi PP: The multilayered complexity of ceRNA crosstalk and competition. Nature 505: 344-352, 2014.
28. Livak KJ and Schmittgen TD: Analysis of relative gene expression data using real-time quantitative PCR and the 2(-Delta Delta C(T)) method. Methods 25: 402-408, 2001

29. Ling C, Wang X, Zhu J, Tang H, Du W, Zeng Y, Sun L, Huang JA and Liu Z: MicroRNA-4286 promotes cell proliferation, migration, and invasion via PTEN regulation of the PI3K/Akt pathway in non-small cell lung cancer. Cancer Med 8: 3520-3531, 2019.

30. Sun Y, Zhu Q, Zhou M, Yang W, Shi H, Shan Y, Zhang Q and Yu F: Restoration of miRNA-148a in pancreatic cancer reduces invasion and metastasis by inhibiting the Wnt/beta-catenin signaling pathway via downregulating maternally expressed gene-3. Exp Ther Med 17: 639-648, 2019.

31. Xu G, Meng L, Yuan D, Li K, Zhang Y, Dang C and Zhu K: MEG3/miR21 axis affects cell mobility by suppressing epithelialmesenchymal transition in gastric cancer. Oncol Rep 40: 39-48, 2018.

32. Long J and Pi X: IncRNA-MEG3 suppresses the proliferation and invasion of melanoma by regulating CYLD expression mediated by sponging miR-499-5p. Biomed Res Int 2018: 2086564, 2018.

33. Su L, Han D, Wu J and Huo X: Skp2 regulates non-small cell lung cancer cell growth by Meg3 and miR-3163. Tumour Biol 37: 3925-3931, 2016.

34. Wang P, Chen D, Ma H and Li Y: lncRNA MEG3 enhances cisplatin sensitivity in non-small cell lung cancer by regulating miR-21-5p/SOX7 axis. Onco Targets Ther 10: 5137-5149, 2017.

35. Zhang J, Yao T, Wang Y, Yu J, Liu Y and Lin Z: Long noncoding RNA MEG3 is downregulated in cervical cancer and affects cell proliferation and apoptosis by regulating miR-21. Cancer Biol Ther 17: 104-113, 2016.

36. Alvarez-Garcia V, Tawil Y, Wise HM and Leslie NR: Mechanisms of PTEN loss in cancer: It's all about diversity. Semin Cancer Biol 59: 66-79, 2019.

37. Papa A and Pandolfi PP: The PTEN-PI3K Axis in Cancer. Biomolecules 9: 153, 2019.

38. Perez-Ramirez C, Canadas-Garre M, Molina MA, Faus-Dader MJ and Calleja-Hernandez MA: PTEN and PI3K/AKT in non-small-cell lung cancer. Pharmacogenomics 16: 1843-1862, 2015.

39. Dong L, Li G, Li Y and Zhu Z: Upregulation of Long Noncoding RNA GAS5 Inhibits Lung Cancer Cell Proliferation and Metastasis via miR-205/PTEN Axis. Med Sci Monit 25: 2311-2319, 2019.

40. Wang H, Ma Z, Liu X, Zhang C, Hu Y, Ding L, Qi P, Wang J, Lu S and $\mathrm{Li}$ Y: MiR-183-5p is required for non-small cell lung cancer progression by repressing PTEN. Biomed Pharmacother 111: 1103-1111, 2019.

41. Yu X, Chen Y, Tian R, Li J, Li H, Lv T and Yao Q: miRNA-21 enhances chemoresistance to cisplatin in epithelial ovarian cancer by negatively regulating PTEN. Oncol Lett 14: 1807-1810, 2017.

42. Fang H, Xie J, Zhang M, Zhao Z, Wan Y and Yao Y: miRNA-21 promotes proliferation and invasion of triple-negative breast cancer cells through targeting PTEN. Am J Transl Res 9: 953-961, 2017.

This work is licensed under a Creative Commons Attribution-NonCommercial-NoDerivatives 4.0 International (CC BY-NC-ND 4.0) License. 